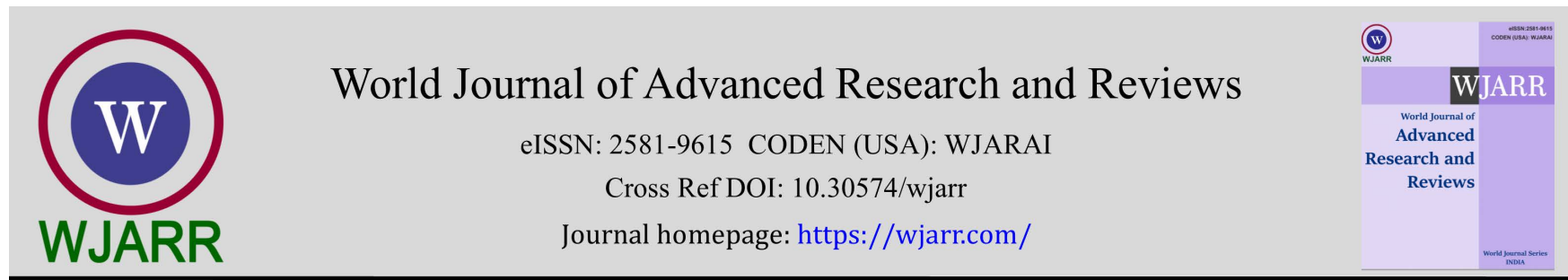

(Review ARticle)

Check for updates

\title{
Angiotensin converting enzyme 2 and inflammatory cytokines: A potential link between chronic periodontitis and COVID-19
}

\author{
Vikas Deo ${ }^{1, *}$, Dinesh Pilania ${ }^{1}$, Pradeep Kumar ${ }^{2}$ and Rajendra Yadav ${ }^{2}$ \\ ${ }^{1}$ Dr S.N Medical College, Jodhpur, India. \\ ${ }^{2}$ Rajasthan Dental College Devi Nagar Jaipur, India.
}

World Journal of Advanced Research and Reviews, 2021, 09(01), 062-068

Publication history: Received on 24 December 2020; revised on 03 January 2021; accepted on 05 January 2021

Article DOI: https://doi.org/10.30574/wjarr.2021.9.1.0498

\begin{abstract}
The global COVID-19 outbreak has caused a public health emergency crisis, as declared by the World Health Organization in January 2020. Periodontitis is associated with increased risk for respiratory condition; hence there is biological plausibility for a link between periodontitis, elevated pro-inflammatory cytokines levels and COVID-19related pulmonary problems. The mechanism could be similar to what has been proposed and established for the progression of COPD in Periodontitis patients. Periodontal pockets have also been found to harbour viral species. The periodontal pockets could provide a favourable environment for SARS-CoV-2 virus replication and survival. ACE2 is a primary receptor for SARS-CoV-2 infections. The oral cavity and lungs are primary targets for SARS-CoV-2 virus. The evidences suggest that the regulation of the inflammatory pattern by ACE2 might also be connected to the response in periodontal disease. ACE2 can regulate the pro-inflammatory and anti-inflammatory processes, balancing cytokine expression. Cytokine storm has been found to be a key feature of COVID-19, where the cytokine levels are very high, inducing an exaggerated general inflammation. The same cytokines are involved in periodontal disease, but they have a different initiation path related to biofilm and bacteria. During a SARS-CoV-2 co-infection, the periodontal disease might be exacerbated due to the down-regulation of ACE2 and an increase in ACE and Ang II, with the consequent involvement of several pro-inflammatory cytokines. Treating any existing periodontal disease and maintaining good periodontal health could prove vital in clinical management of COVID-positive patients.
\end{abstract}

Keywords: Angiotensin Converting Enzyme, Inflammatory Cytokines, Chronic Periodontitis, COVID-19

\section{Introduction}

The global COVID-19 outbreak has caused a public health emergency crisis, as declared by the World Health Organization in January 2020 [1]. While up to $80 \%$ of patients infected by SARSCoV-2 recover after mild cold-like symptoms with no major complications, $20 \%$ can develop serious respiratory complications that can develop into acute respiratory distress syndrome due to the virus' ability to infect human respiratory epithelial cells. Around 5\% of COVID19 patients require intensive care and mechanical ventilation [2].

WHO in its interim guidelines mentioned that while COVID-19 has a viral origin, it is suspected that in severe forms of the infection, bacteria plays a part, increasing the chance of complications such as pneumonia, acute respiratory distress syndrome, sepsis, septic shock and death [3]. Previous studies have linked Periodontitis, a bacterial infection, to various systemic conditions including the respiratory conditions. Individuals with periodontitis have shown increased risk for complications and higher mortality rate in hospitalized patients. Bacteria present in the metagenome of patients severely infected with COVID-19 included high reads for Prevotella, Staphylococcus, and Fusobacterium, all usually commensal organisms of the mouth and are known Periodontal pathogen [4]. There can be a significant impact of a

\footnotetext{
${ }^{*}$ Corresponding author: Vikas Deo

Dr S.N Medical College, Jodhpur.
}

Copyright (C) 2021 Author(s) retain the copyright of this article. This article is published under the terms of the Creative Commons Attribution Liscense 4.0. 
potential connection between periodontal status and Covid-19 given the high prevalence of periodontal disease in adults, the high transmission rate of SARS-CoV-2 and the limited access to periodontal treatment during the pandemic. The aim of the present review is to provide a comprehensive insight into the influence of periodontitis on respiratory health, potential role of periodontal pockets as a reservoir for the virus, role of ACE 2 in inflammatory response, the mechanism of action of key inflammatory cytokines and a possible link between periodontitis and covid-19.

\section{Influence of periodontitis on respiratory health}

The Subgingival micribiota in patients with Periodontitis provides a significant and persistent gram negative bacterial challenge to the host. By genomic sequencing, researchers have identified hundreds of microbial species. Haffajee and Socransky estimated the subgingival biofilms to accommodate more than 700 microbial colonies [5].

In advanced Periodontitis, there is an excessive local inflammatory response to the bacterial stimuli. The disease process involves the loss of epithelial integrity in the periodontal pocket that is, the sulcular epithelium which is frequently ulcerated and discontinuous. The local inflammatory response to the bacterial stimuli and the loss of epithelial integrity in the periodontal pocket allows for penetration of bacteria and their products such as Lippolysaccharides into the inflamed tissues and into the systemic circulation [6]. Hence, patients with untreated Periodontitis have an increased risk for transient bacteraemias. Bacteraemia and associated endotoxemia incite the overproduction of destructive proinflammatory mediators at distant sites in the periodontal patient.

Therefore, patients with Periodontitis may be at greater risk for developing a number of systemic conditions associated with a similar overactive host response to external stimuli, such as cardiovascular disease, adverse pregnancy outcomes and diabetic complications. Elevated levels of cytokines, prostanoids and enzymes are evident in all these conditions.

A relationship between poor periodontal health and respiratory disease, especially in high risk subjects, has been suggested by a number of recent microbiologic and epidemiologic studies. Several reports suggested that potential respiratory pathogens, which cause COPD, colonize the mouth of high risk subjects; for example, those in intensive care unit [7], and nursing home residents [8]. Significantly, results from preliminary trials demonstrated that alteration to oral hygiene significantly reduces the rate of lower respiratory tract infection in institutionalized subjects. These studies suggest that mouth may serve as an important reservoir of lower respiratory tract infection. Studies $[9,10]$ have also suggested an association between poor oral health and COPD, after controlling for other confounding variables such as smoking, gender, age and sex.

A number of mechanisms by which poor oral health may influence the course of COPD can be envisioned. The most direct means by which the oral cavity may influence the lung disease is by aspiration of indigenous oral bacteria in salivary secretions to the lower respiratory tract. Indeed, it is well known that anaerobic lung infections occur following aspiration of salivary secretions, especially in patients with periodontal disease $[11,12]$. Recently, it has been hypothesized that the oral cavity may also influence the oral-pharyngeal colonization by recognized pathogens, especially in high-risk patients with poor oral hygiene. Oral bacteria may stimulate periodontal tissues (epithelial cells, endothelial cells, fibroblasts, macrophages, white cells, peripheral mononuclear cells) to release cytokines such as IL$1 \alpha$, IL-1 $\beta$, IL-6, IL-8 and TNF- $\alpha$ [13]. A mechanism proposed for the gross airway epithelial damage observed in COPD involves cytokines which recruit neutrophils to infiltrate airway parenchyma and to release proteolytic enzymes and toxic oxygen radicals [14]. The release of cytokines from the respiratory epithelium is followed by binding of respiratory pathogens or their products to the respiratory epithelial cells. Oral bacteria in the secretions that adhere to the mucosal surface may bind to mucosal epithelium to stimulate cytokine production. It is also possible that cytokines originating from the oral tissues, for example from the gingival crevicular fluids [14], enters the whole saliva, which then contaminates distal respiratory epithelium. The release of hydrolytic enzymes from these inflammatory cells may result in damage of the epithelium, making it more susceptible to infection by respiratory pathogens.

There is strong evidence that periodontitis is associated with increased risk for respiratory conditions, such as chronic obstructive pulmonary disease (COPD), pneumonia and lung function, hence there is biological plausibility for a link between periodontitis, elevated pro-inflammatory cytokines levels and COVID-19-related pulmonary problems. The mechanism could be similar to what has been proposed and established for the progression of COPD in Periodontitis patients. 


\section{Periodontal pockets as reservoir for virus}

Periodontal pockets have also been found to harbour viral species such as the herpes simplex virus type 1 [15], EpsteinBarr Virus, Human Cytomegalovirus [16] and human papilloma virus [17]. DNA sequencing study of subgingival plaque collected from healthy and periodontal disease patients showed that even though bacteria were the most abundant component of the subgingival plaque, with over $95 \%$ of the reads, viruses were also present in these complex communities associated with bacterial biofilm complexes [18]. Possible sources of initial viral infection of periodontal tissues infection could be as follow: a direct infection of gingival epithelial cells exposed to the oral cavity, virus migration through the blood stream or infected immune cells in the periodontal inflammatory infiltrate [19].

The hypothesis that periodontal pockets could be a favourable environment for SARS-CoV-2 virus replication and survival was explored by Badran et al [20]. These hypothetical niches could act as reservoirs for SARS-CoV-2, whether in an active or latent state. This might be the answer to the re-infection or increasing number of false negative cases being reported as periodontal pockets may act as niches where virus may be harbouring other than the nasopharyngeal/oropharyngeal tracts, where samplings are targeted.

The continuous inflammatory response present in affected sites of periodontal disease could bring mononuclear cells infected by SARS-CoV-2 [21]. Thus, it is reasonable to think that the crevicular fluid on the periodontal pocket could harbour virus arriving from infected mononuclear cells and further mix with patient's saliva.

In order to tackle the COVID-19 pandemic efficiently, it is crucial to reduce the spread of the infection by not only carrying out extraordinary prevention and safety measures, but also identifying possible virus reservoirs in COVIDpositive patients.

\section{Angiotensin converting enzyme 2}

The renin-angiotensin system (RAS) regulates or modulates numerous physiological functions including blood pressure, electrolyte balance, inflammation and the release of various peptide hormones. Prorenin is converted to renin; releasing renin into the circulatory system, thereby cleaving AGT forming angiotensin I (1-10) (Ang I). Ang I can be cleaved by angiotensin I-converting enzyme (ACE) to form angiotensin II (1-8) (Ang II) or by angiotensin I-converting enzyme 2 (ACE-2) to form angiotensin 1-9 (Ang 1-9). ACE-2 also catalyses the cleavage of Ang II to form angiotensin 1-7 (Ang 1-7).

Ang II has a key role in the activation of functions and signalling pathways correlated with inflammation, fibrosis, and tissue injury, such as the generation of free radicals, activation of protein kinases and recruitment of inflammatory cells, and the synthesis and release of cytokines and chemokines [22,23]. ACE2 is a regulator of the RAS system, modulating the damaging actions of Ang II and AT1, decreasing the inflammatory response [22]. ACE and ACE2 are similar enzymes related to contrasting functions.

ACE2 is a primary receptor for SARS-CoV-2 infections. Data from COVID-19 patients have shown an increase in Ang II levels, which was correlated with viral load due to the binding between ACE2 and SARS-CoV-2 [24]. In vivo data on mice infected by SARS-CoV-2 revealed lung failure related to the downregulation of ACE2 [25]. An ACE2-SARS-COV-2 connection could potentially result in reduced ACE2 levels at the cell surface, decreasing the degradation of Ang II, and generating Ang1-7 [26-28]. A reduction in ACE2 through its internalization increases the Ang II/Ang1-7 ratio thereby exacerbating the inflammatory pattern of the SARS-CoV-2 infection. Ang II favours the recruitment of infiltrating inflammatory cells into tissues by stimulating the production of specific cytokines/chemokines such as the potent monocyte chemoattractant protein 1 (MCP-1). Other cytokines involved in the onset and progression of the viral inflammatory condition are tumour necrosis factor alpha (TNF- $\alpha$ ), interleukin-6 (IL-6), and interleukin-1 beta (IL-1 $\beta$ ), inducing apoptosis of endothelial cells and the activation of macrophages in the inflamed tissue or organ. During a SARSCoV-2 infection, Ang II is inefficiently counterbalanced by Ang1-7. The oral cavity and lungs are primary targets for SARS-CoV-2 virus. The oral mucosa and the epithelial cells of the lungs are involved in entrance of the virus, facilitating its replication and inducing an inflammatory response [29].

The initiation or progression of periodontitis also possibly involves a local renin-angiotensin system (RAS) in periodontal tissue. Some studies have demonstrated the existence of local RAS components in oral tissue using cultured guinea pig [30] and rabbit gingival [31] fibroblasts and ferret gingiva [32]. In a landmark study, Santos et al. (2009) [33] demonstrated that a local RAS system is not only present but is also functional in both human and rat periodontal tissue. Furthermore, they also showed that blocking $\mathrm{AT}_{1} \mathrm{R}$ and renin can significantly prevent periodontal bone loss induced 
by EP in rats. Apart from confirming the presence of mRNA for the classic RAS components in both human and rat gingiva, the study demonstrated mRNA expression for angiotensin converting enzyme 2 (ACE-2) and the Mas receptor (MasR). Additionally, ACE activity was also reported to be increased in human gingiva from volunteers with gingivitis. Gabriele et.al (2017) suggested that Angiotensin II Type 1 Receptor knockdown and AT1R pharmacologic blockade by losartan may differently control balance of inflammatory cytokines, such as IL-6 and IL-8, in primary human periodontal fibroblasts [34]. The evidences suggest that the regulation of the inflammatory pattern by ACE2 might be connected to the response in periodontal disease.

\section{Role of ACE 2 in inflammatory response and as a link between periodontitis and covid-19}

Bacteria are involved in facilitating the initiation of an inflammatory response inside the periodontal pocket. ACE2 can regulate the pro-inflammatory and anti-inflammatory processes, balancing cytokine expression through the formation of the ACE2-Ang1-7-MasR axis, which has a primary role in the downregulation of the pro-inflammatory cytokines. Cytokine storm has been found to be a key feature of COVID-19, where the cytokine levels are very high, inducing an exaggerated general inflammation [35]. High IL-6 levels have been linked to significantly higher risk for pulmonary complications and increased need for mechanical ventilation in COVID-19 patients.

The same cytokines are involved in periodontal disease, but they have a different initiation path related to biofilm and bacteria. During a SARS-CoV-2 co-infection, the periodontal disease might be exacerbated due to the down-regulation of ACE2 and an increase in ACE and Ang II, with the consequent involvement of several pro-inflammatory cytokines such as IL-6, TNF- $\alpha$, IL-1 $\beta$, and others. Based upon the increased expression of IL-1, IL- 6 and TNF in inflamed gingiva and high levels in the GCF of periodontitis patients, several studies have suggested that increased production of these cytokines may play an important role in periodontal tissue destruction.

\section{Regulation of Bone Remodeling}

There are 2 molecules considered essential and sufficient to support osteoclastogenesis: macrophage colonystimulating factor (M-CSF or CSF- I) and receptor activator of nuclear factor kappa B ligand (RANKL). M-CSF is a secreted factor but the cell surface form of RANKL requires a juxtacrine (cell to cell) interaction.

The process of bone resorption is initiated with a resorptive stimulus. Osteotrophic factors such as hormones (vitamin D3, parathyroid hormone-related protein), cytokines (interleukin-1, interleukin-6, interleukin-11 and interleukin-17), growth factors (tumor necrosis factor- $\alpha$ and bone morphogenetic protein-2) and other molecules [prostaglandin E2, the T-cell-activating CD40 ligand (CD40L), and glucocoticoid] all enhance expression of the RANKL gene in osteoblasts/stromal cells [36]. All these stimulators typically affect bone resorption through the activation of M-CSF or RANKL. RANKL is also known as osteoprotegerin-ligand, OPG-L, or TRANCE) is a cell surface protein present on osteoblastic cells and is responsible for osteoclast differentiation and bone resorption [37]. RANKL interacts with its receptor, RANK, on hematopoietic cells for differentiation and maturation of osteoclast precursor cells to activate osteoclasts. Osteoprotegerin acts as a decoy receptor, expressed by osteoblastic cells, which binds to RANKL and inhibits osteoclast development. Several studies have shown the opposite effect of RANKL and osteoprotegerin in bone modulation. Moreover, several stimulators of bone resorption that up-regulate RANKL expression inhibit osteoprotegerin expression in osteoblasts/stromal cells [36].

High levels of ACE2 can facilitate anti-inflammatory feedback, binding Ang1-7 and activating MasR. Higher levels of this protein can facilitate the entry of SARS-CoV-2 into the oral cavity [38]. The complex formed between the virus and ACE2 proteins leads to a reduction in ACE2 levels in infected tissue [39], and this might increase the expression of cytokines, with the consequent activation of the osteoprotegerin (OPG)- the receptor for RANK-Ligand (RANK)-RANKL axis, stimulating osteoclast function and improving bone cell phenotype modulation as discussed earlier.

The presence of ACE 2 in periodontitis as well as COVID-19 suggests a possible correlation between them. The up and down regulation of ACE-2 is responsible for activity of various enzymes that play a key role in pathogenesis of both the diseases. Further studies are required to firmly establish this hypothesis which may open new domains for the management of this global pandemic.

\section{Conclusion}

Treating any existing periodontal disease and maintaining good periodontal health could prove vital in clinical management of COVID-positive patients. This will not only help in reducing viral load in the periodontal pockets, but 
would also prevent contribution to the cytokine storm from periodontitis. The relationship between COVID-19 and Periodontitis could even be bi-directional with both these conditions influencing the initiation and progression of each other. Given the similarity in the pathogenesis as evident by common mechanisms such as ACE2 activity, cytokine actions; it is prudent to further explore any such association that may prove beneficial in developing treatment strategies and thereby contributing to overall management of the patients.

Given the high risk of exposure to the examiners, there is dearth of in vivo studies to validate this possible association which, at least in theory, looks plausible. As the understanding about this novel virus improves, more studies, particularly the clinical studies, will prove to be beneficial in not only understanding the possible link between these two diseases, but also to better manage the patients suffering for Covid-19. This might even help in identifying not only better treatment strategies but also help in identifying the individuals at risk for contracting severe form of covid-19 disease.

\section{Compliance with ethical standards}

\section{Acknowledgments}

The authors would like to thank Dr C. S. Chattopadhyay and Dr Dinesh Pilania (Departament of Dentistry, Dr. S. N. Medical College, Jodhpur, India) for help in editing this manuscript.

Sources of funding for the systematic review and other support: None

\section{Disclosure of conflict of interest}

The authors report no conflicts of interest related to this study.

\section{References}

[1] Sohrabi C, et al. World Health Organization declares global emergency: A review of the 2019 novel coronavirus (COVID-19). Int J Surg. 2020; 76: 71-76.

[2] Zhou F, et al. Clinical course and risk factors for mortality of adult inpatients with COVID-19 in Wuhan, China: A retrospective cohort study. Lancet. 2020; 395(10229): P1054-1062 .

[3] World Health Organization. Clinical management of severe acute respiratory infection when COVID-19 is suspected. Interim guidance. 13 March 2020.

[4] Chakraborty S. Metagenome of SARS-Cov2 patients in Shenzhen with travel to Wuhan: OSF Preprints. 2020.

[5] Haffajee AD, Socransky SS. Introduction to microbial aspects of periodontal biofilm communities, development and treatment. Periodontol. 2006; 42: 7-12.

[6] Page RC. The Pathobiology of Periodontal Diseases May Affect Systemic Diseases: Inversion of a Paradigm Ann Periodontol. 1998; 3: 108-120.

[7] Scannapieco FA, Stewart EM, Mylotte JM. Colonization of dental plaque by respiratory pathogens in medical intensive care patients. Crit Care Med. Jun 1992; 20(6): 740-5.

[8] Russell SL, Boylan RJ, Kaslick RS, Scannapieco FA, Katz RV. Respiratory pathogen colonization of the dental plaque of institutionalized elders. Spec Care Dentist. May-Jun 1999; 19(3): 128-34.

[9] Scannapieco FA, Papandonatos GD, Dunford RG. Associations between oral conditions and respiratory disease in a national sample survey population. Ann Periodontol. Jul 1998; 3(1): 251-6.

[10] Scannapieco FA, Wang B, Shiau HJ. Oral bacteria and respiratory infection: effects on respiratory pathogen adhesion and epithelial cell proinflammatory cytokine production. Ann Periodontol. Dec 2001; 6(1): 78-86.

[11] Finegold SM. Aspiration pneumonia. Rev Infect Dis. 1991.

[12] Morris JF, Sewell DL. Necrotizing pneumonia caused by mixed infection with Actinobacillus actinomycetemcomitans and Actinomyces israelii: case report and review. Clin Infect Dis. Mar 1994; 18(3): 4502.

[13] Wilson M, Reddi K, Henderson B. Cytokine-inducing components of periodontopathogenic bacteria. J Periodontal Res. Aug 1996; 31(6): 393-407. 
[14] Birkedal-Hansen H. Role of cytokines and inflammatory mediators in tissue destruction. J Periodontal Res. 1993 Nov 1993; 28(6 Pt 2): 500-10.

[15] Petrović SM, Zelić K, Milasin J, Popović B, Pucar A, Zelić O. Detection of herpes simplex virus type 1 in gingival crevicular fluid of gingival sulcus/periodontal pocket using polymerase chain reaction., Srp Arh Celok Lek. MayJun 2014; 142(5-6): 296-300.

[16] Khosropanah H, Karandish M , Ziaeyan M, Jamalidoust M .Quantification of Epstein-Barr Virus and Human Cytomegalovirus in Chronic Periodontal Patients. Jundishapur J Microbiol. 30 Jun 2015; 8(6): e18691.

[17] Dayakar MM, Shipilova A, Gupta D. Periodontal pocket as a potential reservoir of high risk human papilloma virus: A pilot study J Indian Soc Periodontol. Mar-Apr 2016; 20(2): 136-140.

[18] Dabdoub SM, Ganesan SM, Kumar PS. Comparative metagenomics reveals taxonomically idiosyncratic yet functionally congruent communities in periodontitis. Sci Rep 2016; 6.

[19] Miller CS. Viruses: are they really culprits for periodontal disease? A critical review? J Investig Clin Dent. 2014; 5: 243.

[20] Badran Z, Gaudin A, Struillou X, Amador G, Soueidan A. Periodontal pockets: A potential reservoir for SARSCoV-2? Med Hypotheses. Oct 2020;143.

[21] Li L, Wo J, Shao J, Zhu H, Wu N, Li M, et al. SARS-coronavirus replicates in mononuclear cells of peripheral blood (PBMCs) from SARS patients. J Clin Virol. 2003; 28(3): 239-44.

[22] Capettini, LS, Montecucco F, Mach F, Stergiopulos N, Santos RA, da Silva RF. Role of renin-angiotensin system in inflammation, immunity and aging. Curr. Pharm. Des. 2012; 18: 963-970.

[23] Sukumaran V, Watanabe K, Veeraveedu PT, Veeraveedu PT, Gurusamy N, Ma M, Thandavarayan RA, Lakshmanan AP, Yamaguchi K, Suzuki K. et al. Olmesartan, an AT1 antagonist, attenuates oxidative stress, endoplasmic reticulum stress and cardiac inflammatory mediators in rats with heart failure induced by experimental autoimmune myocarditis. Int. J. Biol. Sci. 2011; 7: 154-167.

[24] Liu Y, Yang Y, Zhang C, Huang F, Wang F, Yuan J, Wang Z, Li J, Li J, Feng C, et al. Clinical and biochemical indexes from 2019-nCoV infected patients linked to viral loads and lung injury. Sci. China Life Sci. 2020; 63: 364-374.

[25] Barton ES, Forrest JC, Connolly JL, Chappell JD, Liu Y, Schnell FJ, Nusrat A, Parkos CA, Dermody TS. Junction adhesion molecule is a receptor for reovirus. Cell. 2001; 104: 441-451.

[26] Yamada Y, Liu DX. Proteolytic activation of the spike protein at a novel RRRR/S motif is implicated infurindependent entry, syncytium formation, and infectivity of coronavirus infectious bronchitis virus in cultured cells. J. Virol. 2009; 83: 8744-8758.

[27] Barton ES, Forrest JC, Connolly JL, Chappell JD, Liu Y, Schnell FJ, Nusrat A, Parkos CA, Dermody TS. Junction adhesion molecule is a receptor for reovirus. Cell. 2001; 104: 441-451.

[28] Santos CF, Morandini AC, Dionísio TJ, Faria FA, Lima MC, Figueiredo CM, Colombini-Ishikiriama BL, Sipert CR, Maciel RP, Akashi AP, et al. Functional local renin-angiotensin system in human and rat periodontal tissue. PLoS ONE 2015, 10, e0134601.

[29] Mancini L, Quinzi V, Mummolo S, Marzo G and Marchetti E. Angiotensin-Converting Enzyme 2 as a Possible Correlation between COVID-19 and Periodontal Disease. Appl. Sci. 2020; 10: 62-24.

[30] Ohuchi N, Koike K, Sano M, Kusama T, Kizawa Y, Hayashi K, et al. Proliferative effects of angiotensin II and endothelin-1 on guinea pig gingival fibroblast cells in culture. Comp Biochem Physiol C Toxicol Pharmacol. 2002; 132: 451-460.

[31] Ohuchi N, Hayashi K, Koike K, Kizawa Y, Kusama T, Ohsawa M, et al. Pharmacological properties of angiotensin II receptors in cultured rabbit gingival fibroblasts. Comp Biochem Physiol C Toxicol Pharmacol. 2004; 137: 281289.

[32] Berggreen E, Heyeraas KJ. Role of K+ATP channels, endothelin A receptors, and effect of angiotensin II on blood flow in oral tissues. J Dent Res. 2003; 82: 33-37.

[33] Santos CF, Morandini AC, Dionísio TJ, Faria FA, Lima MC, Figueiredo CM, Colombini-Ishikiriama BL, Sipert CR, Maciel RP, Akashi AP, Souza GP, Garlet GP, Rodini CO, Amaral SL, Becari C, Salgado MC, Oliveira EB, Matus I, Didier DN, Greene AS. Functional Local Renin-Angiotensin System in Human and Rat Periodontal Tissue. PLoS One. 5 Aug 2015; 10(8): e0134601. 
[34] Gabriele LG, Morandini AC, Dionísio TJ, Santos CF. Angiotensin II Type 1 Receptor Knockdown Impairs Interleukin-1 $\beta$-Induced Cytokines in Human Periodontal Fibroblasts. J Periodontol. Jan 2017; 88(1):e1-e11.

[35] Mehta P, McAuley DF, Brown M, Sanchez E, Tattersall RS, Manson JJ. HLH Across speciality collaboration. COVID19: Consider cytokine storm syndromes and immunosuppression. Lancet. 2020; 395: 1033-1034.

[36] Hofbauer LC, Khosla S, Dunstan CR, Lacey DL, Boyle WJ, Riggs BL. The roles of osteoprotegerin and osteoprotegerin ligand in the paracrine regulation of bone resorption. J Bone Miner Res. Jan 2000; 15(1): 2-12.

[37] Kong YY, Boyle WJ, Penninger JM. Osteoprotegerin ligand: a regulator of immune responses and bone physiology. Immunol Today. Oct 2000; 21(10): 495-502.

[38] Xu H, Zhong L, Deng J, Peng J, Dan H, Zeng X, Li T, Chen Q. High expression of ACE2 receptor of 2019-nCoV on the epithelial cells of oral mucosa. Int. J. Oral Sci. 2020;12: 8.

[39] Silhol F, Sarlon G, Deharo JC, Vaïsse B. Downregulation of ACE2 induces overstimulation of the renin-angiotensin system in COVID-19: Should we block the renin-angiotensin system? Hypertens Res. 2020.

\section{Author's short biography}

Dr Vikas Deo is on the reviewer and editorial board of several national and international
journals. He is also honorary faculty of ADA-CERP. He has over 25 international publications to
his credit. His work has been cited over 500 times till date. He is currently serving as Head of
the Department, Dept of Dentistry, Dr S. N. Medical College, Jodhpur.

